\title{
Shape, size, velocity and field-aligned currents of dayside plasma injections: a multi-altitude study
}

\author{
A. Marchaudon ${ }^{1}$, J.-C. Cerisier ${ }^{2}$, M. W. Dunlop ${ }^{3}$, F. Pitout ${ }^{4}$, J.-M. Bosqued $^{5}$, and A. N. Fazakerley ${ }^{6}$ \\ ${ }^{1}$ Laboratoire de Physique et Chimie de l'Environnement et de l'Espace, CNRS et Université d'Orléans, 3A avenue de la \\ Recherche Scientifique, 45071 Orléans Cedex 2, France \\ ${ }^{2}$ Laboratoire de Physique des Plasmas, Ecole Polytechnique, CNRS et Université Pierre et Marie Curie-Paris 6, 4 avenue de \\ Neptune, 94107 Saint-Maur-des-Fossés Cedex, France \\ ${ }^{3}$ Rutherford-Appleton Laboratory, Chilton, Didcot, Oxon, OX11 0QX, UK \\ ${ }^{4}$ Laboratoire de Planétologie de Grenoble, CNRS et Université de Grenoble 1, BP 53, 38041 Grenoble Cedex 9, France \\ ${ }^{5}$ Centre d'Etude Spatiale des Rayonnements, CNRS et Université de Toulouse 3, 9 avenue du Colonel Roche, 31028 Toulouse \\ Cedex 4, France \\ ${ }^{6}$ Mullard Space Science Laboratory, University College London, Holmbury St Mary, Dorking, RH5 6NT, Surrey, UK
}

Received: 29 September 2008 - Revised: 6 February 2009 - Accepted: 18 February 2009 - Published: 12 March 2009

\begin{abstract}
On 20 February 2005, Cluster in the outer magnetosphere and Double Star-2 (TC-2) at mid-altitude are situated in the vicinity of the northern cusp/mantle, with Cluster moving sunward and TC-2 anti-sunward. Their magnetic footprints come very close together at about 15:28 UT, over the common field-of-view of SuperDARN radars. Thanks to this conjunction, we determine the velocity, the transverse sizes, perpendicular and parallel to this velocity, and the shape of three magnetic flux tubes of magnetosheath plasma injection. The velocity of the structures determined from the Cluster four-spacecraft timing analysis is almost purely antisunward, in contrast with the antisunward and duskward convection velocity inside the flux tubes. The transverse sizes are defined from the Cluster-TC-2 separation perpendicular to the magnetic field, and from the time spent by a Cluster spacecraft in one structure; they are comprised between 0.6 and $2 R_{E}$ in agreement with previous studies. Finally, using a comparison between the eigenvectors deduced from a variance analysis of the magnetic perturbation at the four Cluster and at TC-2, we show that the upstream side of the injection flux tubes is magnetically well defined, with even a concave front for the third one giving a bean-like shape, whereas the downstream side is far more turbulent. We also realise the first quantitative comparison between field-aligned currents at Cluster calculated with the curlometer technique and with the single-spacecraft method, assuming infinite parallel cur-
\end{abstract}

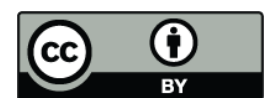

Correspondence to: A. Marchaudon (aurelie.marchaudon@cnrs-orleans.fr) rent sheets and taking into account the velocity of the injection flux tubes. The results agree nicely, confirming the validity of both methods. Finally, we compare the field-aligned current distribution of the three injection flux tubes at the altitudes of Cluster and TC-2. Both profiles are fairly similar, with mainly a pair of opposite field-aligned currents, upward at low-latitude and downward at high-latitude. In terms of intensity, the field-aligned currents at Cluster are two to three times less intense than at TC-2 for the first two flux tubes, in agreement with magnetic field line convergence. For the third flux tube, the intensity is equal, which is explained by the fact that TC-2 crosses the tube on its edge. Finally, the analysis of the ion and electron moments at Cluster shows that the field-aligned currents result from a small difference between upward ion and electron fluxes.

Keywords. Magnetospheric physics (Current systems; Magnetopause, cusp, and boundary layers; Plasma convection)

\section{Introduction}

Pulsed magnetic reconnection known as Flux Transfer Event (FTE) at the magnetopause and direct plasma injection in the polar cusp are responsible for a large part of mass and momentum transfer from the solar wind to the magnetosphere. FTEs formed on the magnetopause are dragged by the magnetic tension at the reconnection point and by the solar wind flow, successively across the Low-Latitude Boundary Layer

Published by Copernicus Publications on behalf of the European Geosciences Union. 
(LLBL) (Eastman et al., 1976), the polar cusp and the plasma mantle region (Rosenbauer et al., 1975) where they eventually disappear.

The transverse size of FTEs at the magnetopause has been extensively studied during the early years following their discovery (Russell and Elphic, 1978; Saunders et al., 1984; Rijnbeek et al., 1984) with values between 1 and $2 R_{E}$. The capabilities of the four-Cluster spacecraft mission have recently allowed refined studies of FTE size from 0.3 to $2 R_{E}$, together with estimations of their velocity varying from 70 to $200 \mathrm{~km} \mathrm{~s}^{-1}$ at the magnetopause (Owen et al., 2001; Bosqued et al., 2001; Sonnerup et al., 2004; Hasegawa et al., 2006). In the high-altitude cusp, the transverse size of reconnected flux tubes has also been estimated with Cluster in the range $700-1500 \mathrm{~km}$ (for the longitudinal extent) with velocities of the tubes between 7 and $20 \mathrm{~km} \mathrm{~s}^{-1}$ during quiet IMF conditions, in agreement with ionospheric conjugate SuperDARN measurements (Vontrat-Reberac et al., 2003; Marchaudon et al., 2004a). In the ionosphere, the size of Pulsed Ionospheric Flows (PIFs) or Poleward Moving Auroral Forms (PMAFs), recognized as signatures of pulsed magnetopause reconnection detected, respectively, from radars and from all-sky optical cameras, has also been extensively studied and is compatible with the size of magnetopause FTEs mapped into the ionosphere (Sandholt et al., 1990; Lockwood et al., 1990; Provan et al., 1998; Milan et al., 2000). An important finding of these ground-based observations is that the latitudinal extent of these signatures $(50-400 \mathrm{~km})$ is in general narrower than their longitudinal extent $(200-2000 \mathrm{~km})$. By contrast, in the magnetosphere a purely cylindrical or elliptical transverse shape has almost always been assumed, apart from Sonnerup et al. (2004) who used a Grad-Shafranov reconstruction with Cluster data to obtain a map of a FTE cross section at the magnetopause, showing its irregular shape.

Many electrodynamic models of Field-Aligned Current (FAC) distributions associated with FTEs have been proposed, such as Lee (1986) with a coaxial distribution, upward current at the tube centre and downward current on its surface, or Southwood (1987) with a pair of opposite currents flowing on each side of the tube with respect to its travelling axis. Cowley and Lockwood (1992) have also suggested a global dayside electrodynamic model, where patches of newly open flux created by reconnection push the open-closed boundary (OCB) equatorward and then move into the polar cap under the magnetic tension effect at the reconnection site and the solar wind flow. In this model, a pair of opposite FACs also flows on each side of the new open flux region. Observations during the past twenty years have strongly favoured pairs of opposite FACs flowing on the FTE sides and closing in the ionosphere at least partly through a Pedersen current inside the FTE. These results were obtained from comparisons between modelling and satellite data (Basinska et al., 1989; Escoubet et al., 1992), from ground-based camera and radar correlated observations (e.g. Milan et al., 2000; Oksavik et al., 2004) or from di- rect single-spacecraft field-aligned current estimation in association with ground-based observations (Marchaudon et al., 2004b; Bosqued et al., 2005). However, recent studies (Rinne et al., 2007) have shown that, during $B_{y}$ dominating Interplanetary Magnetic Field (IMF), FTE electrodynamics could be more complex, with an asymmetric twin-cell flow pattern for which Moen et al. (2008) suggested two interpretations, either in terms of two independent magnetosphereionosphere current loops or in terms of a non-negligible parallel electric field.

Because the Cluster trajectory is spanning higher-altitude regions than previous missions, recent studies have described magnetosheath injections on the tailward side of the cusp and in the mantle. They were associated with lower-altitude observations, including ionospheric polar cap patches detected by the EISCAT Svalbard Radar (ESR) by Lockwood et al. (2001b), PIFs seen by SuperDARN radars and cusp ionsteps observed onboard FAST by Farrugia et al. (2004) and Lund et al. (2008). Lockwood et al. (2001b) interpreted these injections as fossil remnants of a series of magnetopause reconnection pulses. Lund et al. (2008) showed that momentum transfer can still occur on these high-latitude injections via Alfvén waves on old open field lines. In parallel, dayside auroral observations from all-sky cameras have shown different steps in PMAFs activity during dominant dawn-dusk interplanetary magnetic field (IMF- $B_{y}$ ) periods (Sandholt et al., 2004; Sandholt and Farrugia, 2007a, b). In association with SuperDARN convection maps and low-altitude satellite passes, these authors also determined the electrodynamic pattern of the last phase of these PMAFs, characterised by a pair of opposite field-aligned currents called $\mathrm{C} 1 / \mathrm{C} 2$ whose polarity depends upon the sign of the IMF- $B_{y}$ component. These results were consistent with the low-latitude cleft current/high-latitude cleft current (LCC/HCC) distribution initially defined by Taguchi et al. (1993). All these observations are in agreement with the reactivation mechanism of old open flux by the solar wind-magnetosphere dynamo, as initially proposed by Stern (1984). They are also in agreement with the idea of two different steps in the lifetime of magnetosheath injections (or FTEs). The first step corresponds to newly reconnected field lines mainly triggered by magnetic tension at the reconnection site with intense dayside magnetosheath precipitation and associated auroral emissions. The second step called fossil corresponds to older reconnected field lines reactivated by solar wind flow with lower magnetosheath precipitation and ionospheric convection bursts (Cerisier et al., 2005).

On 20 February 2005, during a period of $B_{y}$ dominated IMF, Cluster at high-altitude and Double Star-2 (TC-2) at mid-altitude are situated in the plasma mantle in the vicinity of the northern cusp, with Cluster moving sunward and TC2 anti-sunward. Their magnetic footprints come very close together at about 15:28 UT, over the common field-of-view of SuperDARN radars. Based on this conjunction, we investigate in detail the transverse structure (velocity, size, and 
shape) and the field-aligned current distribution (pattern and intensity at different altitudes, particle carriers) associated with old plasma injections from the magnetosheath, during the fossil second step of their lifetime.

\section{Instrumentation}

Cluster consists in four identical satellites on an elliptical polar orbit with a perigee of $\sim 5 R_{E}$, an apogee of $\sim 20 R_{E}$ and a period of $\sim 58 \mathrm{~h}$. Double Star- 2 has also an elliptical polar orbit with a perigee of $\sim 1.1 R_{E}$, an apogee of $\sim 7 R_{E}$ and a period of $\sim 12 \mathrm{~h}$. Part of the TC-2 instrumentation is very similar to the Cluster instrumentation.

Onboard Cluster, the Plasma Electron and Current Experiment (PEACE) (Johnstone et al., 1997) provides the electron velocity distribution function every $\sim 4 \mathrm{~s}$ (spacecraft spin period), in the energy range from $\sim 1 \mathrm{eV}$ to $\sim 26 \mathrm{keV}$. PEACE is composed of a Low-Energy Electron Analyser (LEEA) and a High-Energy Electron Analyser (HEEA) located on opposite sides of the spacecraft, which measure overlapping energy ranges. In burst mode, the full three-dimensional (3-D) distribution is available and the resulting density and velocity moments are calculated on the ground with a resolution up to $\sim 4 \mathrm{~s}$. Onboard TC-2, only one sensor (LEEA design) of the PEACE experiment is installed. This sensor samples successively a low energy band during a first spin then a high energy band during a second spin, so that data from the full energy range is transmitted during a pair of spins, providing the full three-dimensional (3-D) distribution with a $8 \mathrm{~s}$ resolution (Fazakerley et al., 2005).

The Cluster Ion Spectrometry (CIS) experiment consists in two different instruments: the Hot Ion Analyser (HIA) and the COmposition and DIstribution Function analyser (CODIF) (Rème et al., 2001). Both instruments were switched off on sc-2 so as HIA on sc-4. For this study, we use HIA on sc-1 and sc-3 which offers a good energy and angular resolution without mass resolution and provides the full three-dimensional energy/velocity distribution (assuming all ions are protons) from thermal energies up to about $32 \mathrm{keV} \mathrm{q}^{-1}$. Since HIA is not available on sc-4, we use CODIF which offers a medium angular resolution but measures the full three-dimensional energy/velocity distribution of the major magnetospheric ions $\left(\mathrm{H}^{+}, \mathrm{He}^{+}, \mathrm{He}^{++}\right.$and $\left.\mathrm{O}^{+}\right)$ up to about $38 \mathrm{keV} \mathrm{q}^{-1}$. Only the proton distribution is used in this case. The density and velocity moments are calculated onboard with a $\sim 4 \mathrm{~s}$ resolution. There is no CIS experiment onboard TC-2.

Onboard Cluster and TC-2, the Flux Gate Magnetometer (FGM) (Balogh et al., 2001; Carr et al., 2005) measures the 3 -D magnetic field vector, with up to $\sim 202$ vectors $\mathrm{s}^{-1}$ sampling. We use data averaged to $4 \mathrm{~s}$ resolution.

During this event, the four Cluster spacecraft are in a regular tetrahedral configuration with a $\sim 1000 \mathrm{~km}$ inter-satellites separation and are operating in burst mode, which allows ac-

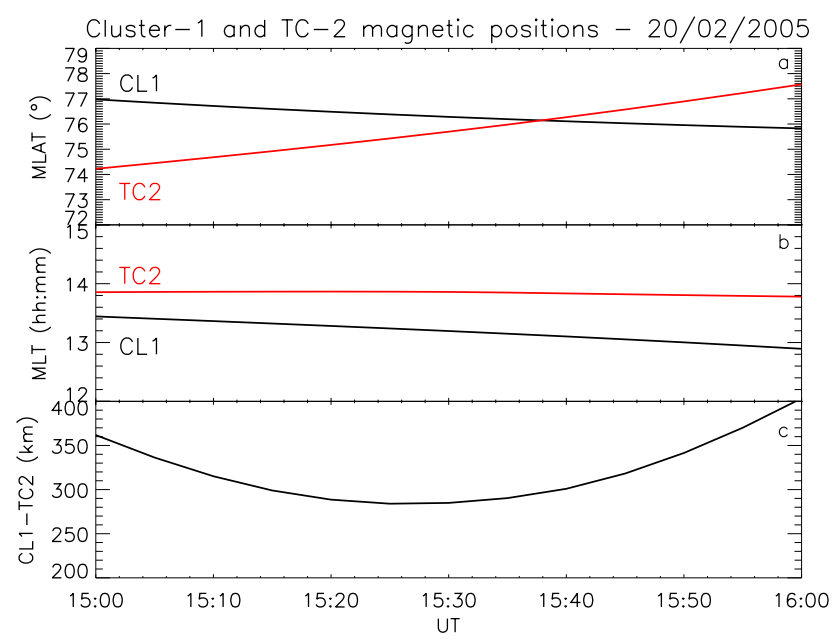

Fig. 1. Cluster-1 (in black) and TC-2 (in red) positions for the period 15:00-16:00 UT. (a) Magnetic (AACGM) latitude (MLAT). (b) Magnetic local time (MLT). (c) Transverse distance between Cluster-1 and TC-2, mapped at the ionospheric altitude $(200 \mathrm{~km})$.

curate evaluations of the current density by the curlometer technique and from particle moments.

In the conjugate ionosphere, SuperDARN radars (Greenwald et al., 1995) measure the line-of-sight (1-o-s) velocity of the ionospheric plasma in 16 adjacent beam directions separated by $3.3^{\circ}$ in azimuth. A full scan is completed in 2 min and thus covers $53^{\circ}$ in azimuth and over $3000 \mathrm{~km}$ in range with a resolution of $45 \mathrm{~km}$. For this event, data of the Stokkseyri, Goose Bay, Kapuskasing and Saskatoon radars are used to reconstruct the dayside ionospheric convection flow patterns, by the "map potential technique" developed by Ruohoniemi and Baker (1998).

Finally, solar wind plasma and interplanetary magnetic field data are obtained from the Solar Wind Electron Proton Alpha Monitor (SWEPAM) and the Magnetic Field Experiment (MAG) on the ACE satellite. These data are used for monitoring the interplanetary medium, with the appropriate lag to take into account the solar wind propagation delay from ACE to Earth.

\section{General context}

Between 14:00 and 16:00 UT on 20 February 2005, the solar wind bulk speed measured by ACE is $\sim 435 \mathrm{~km} \mathrm{~s}^{-1}$, leading to a solar wind propagation delay to the Earth of $\sim 53 \mathrm{~min}$. At Earth, from 14:55 to 15:55 UT, the 3 components of the lagged interplanetary magnetic field are stable, with IMF- $B_{x}$ oscillating between -1.5 and $-2.5 \mathrm{nT}$, a dominating IMF- $B_{y}$ oscillating between -4 and $-4.5 \mathrm{nT}$ and IMF- $B_{z}$ oscillating between -3 and $-3.5 \mathrm{nT}$ (not shown). The lagged solar wind plasma parameters are also stable with a solar wind dynamic pressure of $\sim 1.8 \mathrm{nPa}$. 


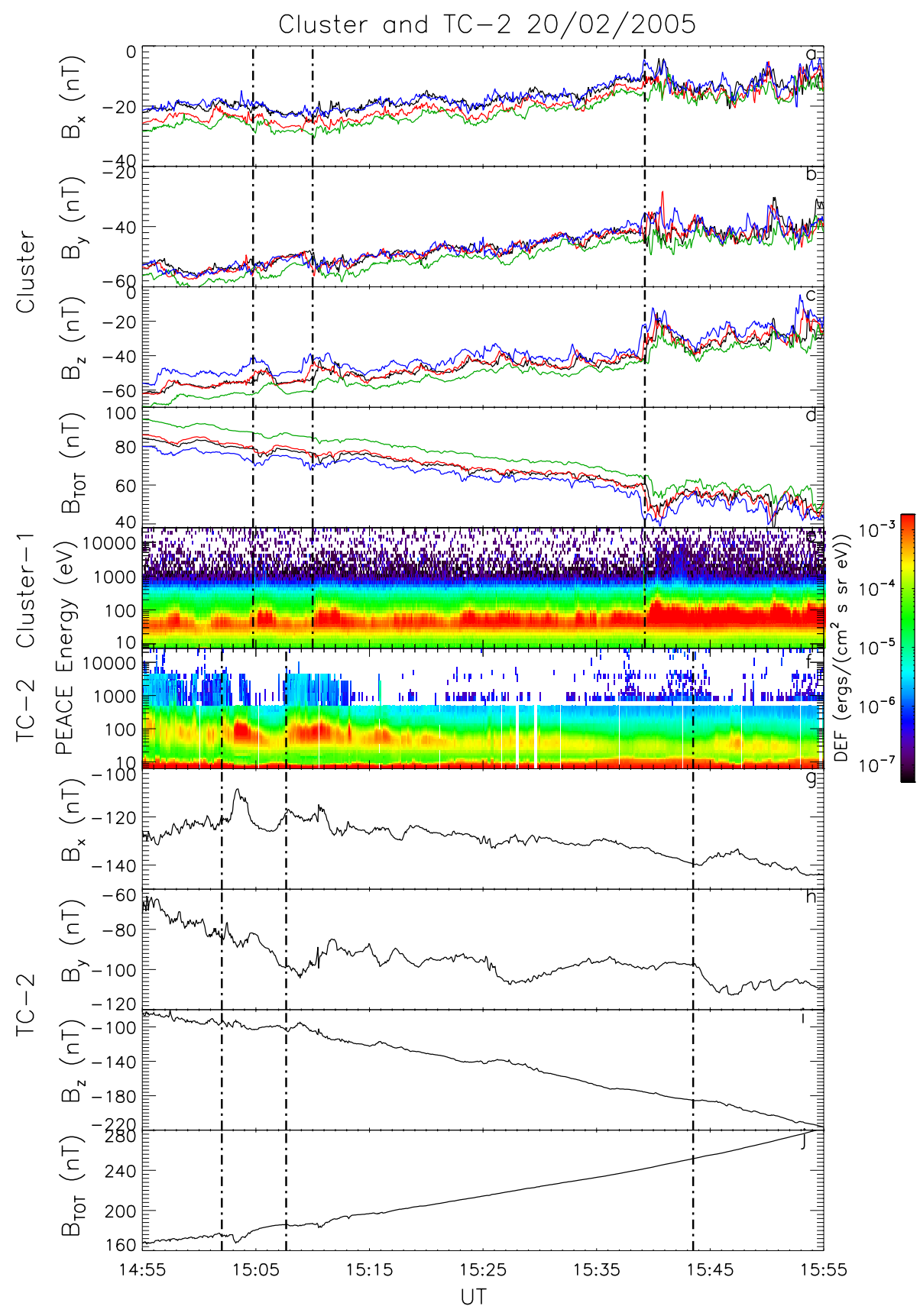

Fig. 2. Overview of the Cluster and TC-2 magnetic field (FGM) and electron fluxes (PEACE) for the 14:55-15:55 UT period. (a) to (d) $X$, $Y, Z$ components and magnitude of the magnetic field at the four Cluster in GSM coordinates (black line for sc-1, red for sc-2, green for sc-3 and blue for sc-4). (e) and (f) Omnidirectionnal electron energy-time spectrograms at Cluster-1 and TC-2. (g) to (j) $X, Y, Z$ components and magnitude of the magnetic field at TC-2 in GSM coordinates. The dash-dotted lines represent the beginning of each injection.

During this period, Cluster and TC-2 are in a favourable magnetic conjunction close to the northern exterior cusp on its afternoonside. At 15:30 UT, Cluster-1, located at $\sim 9 R_{E}$ from the Earth $\left(\sim 4.7, \sim 2.0, \sim 7.4 R_{E}\right.$, GSM coordinates) travels sunward. At the same time, TC-2, located at $\sim 6.1 R_{E}$ from the Earth $\left(\sim 3.0, \sim 1.3, \sim 5.2 R_{E}\right)$ travels essentially antisunward. The magnetic coordinates of both spacecraft and their relative distance mapped to the ionosphere with the Tsyganenko T01 field model (Tsyganenko, 2002a, b) are shown on Fig. 1. The closest approach between Cluster and TC-2 occurs around 15:28 UT at $\sim 75.5^{\circ}$ MLAT and $\sim 13.6$ MLT in Altitude Adjusted Corrected Geo-Magnetic coordinates (AACGM). 

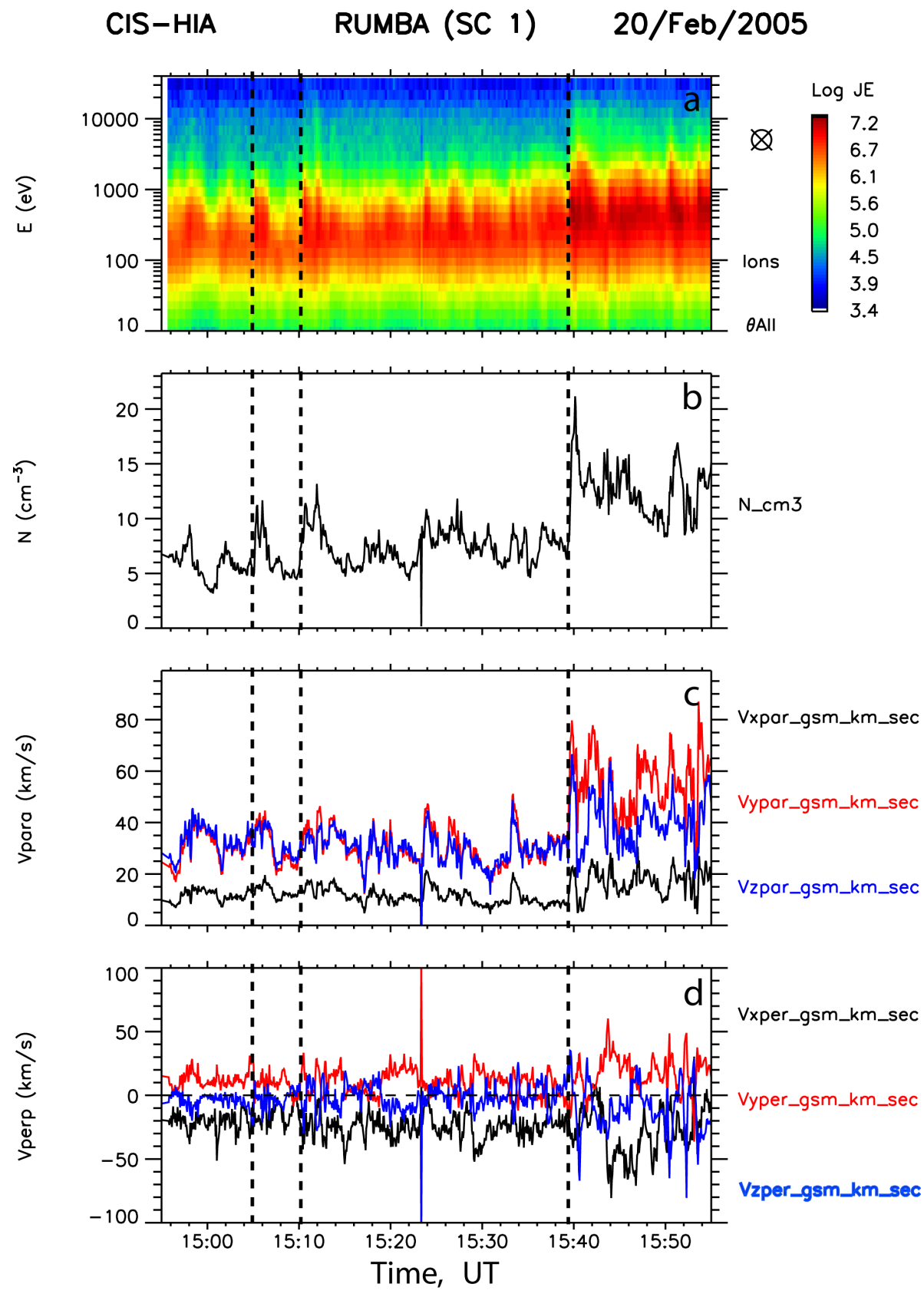

Fig. 3. Overview of the CIS-HIA data of Cluster-1 for the period 14:55-15:55 UT. (a) Omnidirectional ion spectrogram. (b) ion density. (c) and (d) $X, Y, Z$ components of the parallel ion velocity and of the perpendicular ion velocity in GSM coordinates. The dashed lines represent the beginning of each injection.

An overview of magnetic field data and omnidirectionnal electron spectrograms of Cluster-1 and TC-2 between 14:55 and 15:55 UT is presented on Fig. 2. Cluster-1 ion data for the same period are shown on Fig. 3. The ion spectrogram of Cluster-1 (panel a of Fig. 3) and the electron spectrograms of Cluster-1 and TC-2 (panels e and f of Fig. 2) display magnetosheath-like plasma injections, but with a high energy limit slightly lower than generally observed in cusp plasma injections especially for the ions. High-energy electrons characteristic of closed magnetospheric field lines are also observed in the anti-parallel direction on several of the injection signatures. This mixing of magnetospheric and magnetosheath plasmas inside the magnetosphere is most likely caused by dayside magnetic reconnection. Each plasma signature is also accompanied by a drop of the magnetic field magnitude (panels d and j of Fig. 2), indicating a diamagnetic 


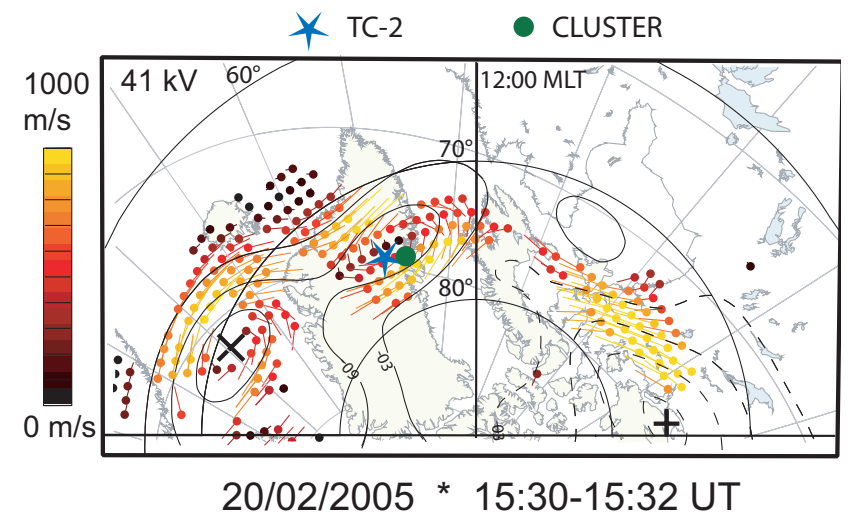

Fig. 4. SuperDARN convection map at 15:30-15:32 UT in AACGM coordinates. The black solid lines represent the equipotential contours and the coloured dots represent the grid points where the convection vectors are determined. The green dot and the blue star represent the Cluster-1 and TC-2 positions.

effect and shows significant magnetic gradients on its edges. The component of the Cluster electron and ion bulk velocities aligned to the magnetic field is upward during the whole period of interest and it exhibits an upward acceleration during the injections (panel c of Fig. 3). This suggests that these injections are initiated on the dayside magnetopause and then convected towards the polar cleft/cusp and mantle where a majority of ions has already mirrored. The presence of electrons results from the need for quasi-neutrality. These observations are in agreement with statistical observations of upward ion flow on the tailward edge of the high-altitude cusp made by Lavraud et al. (2005). Finally, ion convection velocities are essentially in the antisunward and duskward direction (panel d of Fig. 3), in agreement with the expected polar cap flow for the prevailing IMF orientation (negative IMF$B_{y}$ and $-B_{z}$ ). These observations suggest that Cluster and TC-2 are located in the tail lobe on the dusk edge of the cusp proper. Moreover, each plasma signature is observed successively on the four Cluster spacecraft, confirming that these signatures are drifting plasma injections and not successive back and forth motions of the magnetopause boundary layer, tailward of the cusp. Cluster low-energy magnetosheath injections similar to those presented above have already been described by Lockwood et al. (2001b), Farrugia et al. (2004) and Lund et al. (2008). They were observed even more tailward than in our event and were identified as old open flux tubes reactivated by the solar wind-magnetosphere dynamo, as already mentioned in the introduction. Thus, the magnetosheath injections presented in this paper appear as old injections observed in the second step of their lifetime.

From electron spectra such as those displayed on Fig. 2, an identification by eye can be made between similar injections seen at Cluster-1 and TC-2. Three main injections are identified around 15:05, 15:10 and 15:39 UT, Cluster start time (15:02, 15:08 and 15:43 UT, TC-2 start time), and numbered in the following 1, 2, and 3. TC-2 detects Injections 1 and 2 several minutes before Cluster and Injection 3 several minutes after Cluster, setting approximately the time of simultaneous observations around 15:20 UT. A more precise method to estimate the closest conjunction between Cluster and TC2 will be presented in Sect. 4.2, confirming accurately our initial identification of the injections at both spacecraft.

During the whole period of interest, SuperDARN global convection maps show the typical dayside convection cells for negative IMF- $B_{y}$ and $-B_{z}$, with a crescent cell at dusk and a circular cell at dawn, and antisunward and duskward plasma flow in the polar cap. These observations are in agreement with the Cluster ion convection velocities. The SuperDARN convection map at 15:30-15:32 UT, between Injections 2 and 3 is displayed on Fig. 4. Cluster and TC-2 footprints (green dot and blue star) are located downstream of the region of plasma entry in the polar cap, close to the convection reversal boundary (CRB) of the dusk crescent cell. The relative position of Cluster and TC-2 footprints with respect to the CRB will be discussed in the next section.

\section{Velocity and structure of injection flux tubes}

\subsection{Velocity analysis}

We have analysed independently the motion of flux tubes and the convection of the plasma inside these flux tubes in order to emphasize the difference between these two motions.

\subsubsection{Velocity of injection flux tubes}

At Cluster, the velocity of flux tubes can be determined from the delays in the observation of similar signatures at the four spacecraft (four-spacecraft timing analysis). We have used magnetic signatures from the FGM experiment which provide a good time resolution and reflect both diamagnetic effects due to plasma injections and signatures of parallel currents. In this analysis, a quasi-planar front moving across the Cluster tetrahedron has to be assumed. We will show in Sect. 4.4 that this assumption is valid.

The flux tubes velocity at Cluster associated with the three identified injections (at 15:05, 15:10 and 15:39 UT, Cluster time) is shown in the second column of Table 1 (modulus and unit vector in GSM coordinates). For all three injections, the velocity is essentially in the antisunward direction, with a significant increase in the modulus for Injection 3. This latter variation reflects more likely the motion of Cluster approaching the cusp on its afternoon side from the polar cap than temporal effects related to interplanetary drivers since the IMF and solar wind parameters are stable during the period. 
Table 1. Velocity of injections flux tubes and convection velocity at Cluster and mapped into the ionosphere along magnetic field lines and along the SuperDARN Stokkseyri radar beam with which Cluster is magnetically conjugated.

\begin{tabular}{|c|c|c|c|c|}
\hline \multirow{2}{*}{$\begin{array}{l}\text { Event } \\
\text { (Cluster time, UT) }\end{array}$} & \multicolumn{2}{|c|}{ Cluster velocity $\left(\mathrm{km} \mathrm{s}^{-1}\right)$ and direction (GSM xyz) } & \multicolumn{2}{|c|}{ Cluster convection velocity mapped to the ionosphere $\left(\mathrm{m} \mathrm{s}^{-1}\right)$} \\
\hline & Flux tube velocity & Convection velocity & $\begin{array}{l}\text { Convection } \\
\text { velocity }\end{array}$ & $\begin{array}{l}\text { Component along the radar beam } \\
\qquad>0 \text { towards the radar })\end{array}$ \\
\hline Injection $1(15: 05)$ & $26.2(-0.96-0.160 .25)$ & $19.4(-0.870 .47-0.14)$ & 680 & +590 \\
\hline Injection $2(15: 10)$ & $21.5(-0.920 .140 .36)$ & $25.0(-0.900 .43-0.09)$ & 860 & +690 \\
\hline Injection $3(15: 39)$ & $39.4(-0.96-0.040 .29)$ & $32.6(-0.790 .51-0.33)$ & 1250 & +1060 \\
\hline
\end{tabular}

\subsubsection{Convection velocity}

The convection velocity measured by the CIS experiment (panel d of Fig. 3) during the selected injection events is given in column 3 of Table 1 . As compared with the flux tubes velocity, it shows also antisunward but larger duskward components. This difference supports the idea that the velocity of the flux tubes and the convection velocity inside are not equal for this event. These convection velocities, together with the Cluster positions have been mapped along magnetic field lines to the ionosphere (column 4 in Table 1), using the Tsyganenko T01 magnetic field model. During this event, Cluster was magnetically conjugate with the Stokkseyri SuperDARN radar. Because of the inaccuracy of the Tsyganenko model at the Cluster position (see Sect. 4.2 below), the precision of the field line mapping does not exceed one or two radar cells. Anyhow, the radar data confirm clearly that Cluster is situated poleward of the convection reversal boundary (CRB) which marks the transition between antisunward convection in the polar cap and sunward convection in the auroral zone. For the velocity mapping, equipotential field lines have been assumed as suggested by the absence in the PEACE data of large-scale inverted V structures able to affect the large-scale convection (Kullen et al., 2008). During Injections 1 and 2, the mapped Cluster convection velocity component along the radar beam is $590 / 690 \mathrm{~m} \mathrm{~s}^{-1}$ (column 5 of Table 1). These values compare well with the SuperDARN radial velocity of $500 \mathrm{~m} \mathrm{~s}^{-1}$ (a mean on two beams and two range cells to take into account the uncertainty in the mapping) measured in the appropriate radar cells at the same time. However during Injection 3, the noisy radar data do not allow an accurate measurement of the velocity for comparison. These ionospheric observations support that Cluster and TC-2 are not in the cusp proper, but in the plasma mantle region, poleward and duskward of the polar cusp (Newell and Meng, 1992), where the convection is similar in direction (antisunward and duskward) but slower than in the cusp proper.

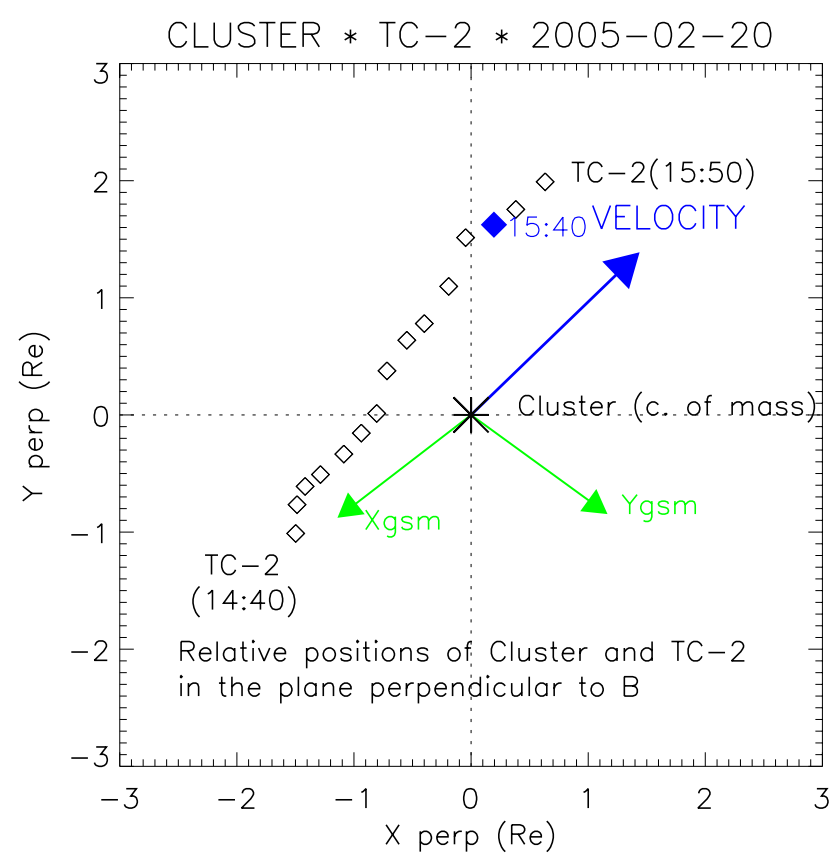

Fig. 5. Relative position of TC-2 with respect to Cluster centre of mass, in the plane perpendicular to the magnetic field for the 14:4015:50 UT period. TC-2 position has been mapped at the Cluster altitude. The $X$ and $Y$ axis of the GSM frame have been projected onto this plane (green arrows). The blue diamond shows the position of TC- 2 at the time of Injection $3(\sim 15: 40$ UT). The blue arrow represents the velocity of Injection 3.

\subsection{Analysis of the Cluster-TC-2 conjunction}

The visual analysis of the electron (and ion) spectrograms on Cluster and TC-2 led us to set the period of correlated observations between 15:00 and 15:50 UT. From Fig. 1, the two spacecraft are situated at the same magnetic latitude at 15:35 UT, with a separation of $28 \mathrm{~min}$ in MLT. Their closest approach (across the field lines) occurs a few minutes earlier around 15:28 UT. However, in the outer cusp, the magnetic field model Tsyganenko (T01) used for calculating the conjunction parameters is only a rough approximation to the real field. A discrepancy between the Tsyganenko model and 
Table 2. Delay from Cluster to TC-2 and size of plasma injections at Cluster.

\begin{tabular}{lcccc}
\hline Event (Cluster time, UT) & \multicolumn{2}{c}{$\begin{array}{c}\text { Delay between Cluster and TC-2 (s) } \\
\text { Calculated }\end{array}$} & $\begin{array}{c}\text { Minimum transverse size } \\
\text { Of the structure }\left(R_{E}\right)\end{array}$ & $\begin{array}{c}\text { Observed duration (s) and longitudinal } \\
\text { size of the structure }\left(R_{E}\right)\end{array}$ \\
\hline Injection 1 (15:05) & -200 & -165 & 0.65 & $150 \mathrm{~s}-0.6 R_{E}$ \\
Injection 2 (15:10) & -170 & -140 & 0.6 & $230 \mathrm{~s}-0.8 R_{E}$ \\
Injection 3 (15:39) & +205 & +255 & 1.0 & $300 \mathrm{~s}-1.9 R_{E}$ \\
\hline
\end{tabular}

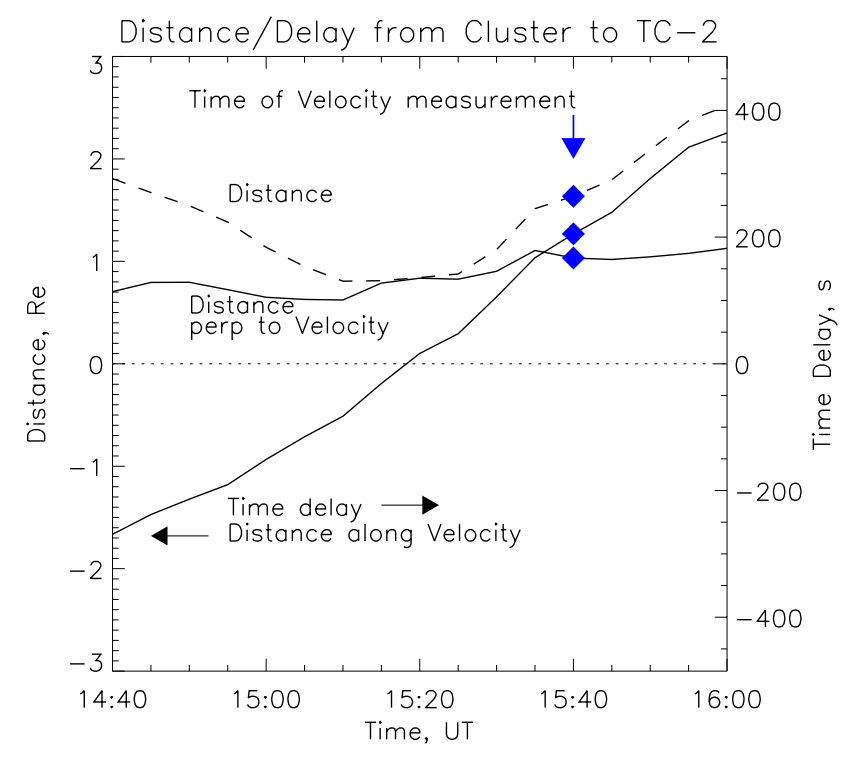

Fig. 6. Components of the relative TC-2 position vector along and transverse to the velocity of Injection 3 (blue diamond) and mapped at the Cluster altitude (solid lines). The dotted line shows the mapped distance between the two spacecraft. The scale on the right gives the expected delay between observations at Cluster and TC-2.

the measured field components reaching 50\% is observed at the Cluster position. So, we have recalculated the field line through TC-2 and up to the Cluster altitude with a "fitted" field obtained by adding the discrepancy observed at Cluster to the Tsyganenko model. Figure 5 shows the relative position of the two spacecraft (with the centre of mass of Cluster at the origin of coordinates) in the plane perpendicular to the magnetic field. The irregular trajectory of TC-2 in this diagram reflects the fluctuations of the magnetic field direction at Cluster. The direction of the velocity observed at the time of Injection 3 (blue diamond) is also indicated.

Figure 6 shows the two components of the relative TC2 position vector, along and transverse to the velocity determined for Injection 3. Knowing this velocity, the longitudinal component gives also an evaluation of the expected delay between the observation of similar features at Cluster and TC-2. For each of the three injections observed between
15:00 and 15:50 UT, we have calculated the expected delay between Cluster and TC-2, based on their velocity given in column 2 of Table 1. Column 2 of Table 2 gives the result of these evaluations for the three injections and column 3 gives the delays identified from observation of the Cluster and TC-2 PEACE spectrograms. For all three injections, the fairly good agreement between the two determinations confirms unambiguously the initial association made by eye.

\subsection{Transverse size of injection flux tubes at Cluster}

As seen on Fig. 6, a minimum transverse size (with respect to their velocity) of injection flux tubes can been deduced from the transverse separation between Cluster and TC-2. Column 3 of Table 2 gives the result of this evaluation for the three injections observed at Cluster, at 15:05, 15:10 and 15:39 UT. They are comprised between 0.6 and $1.0 R_{E}$. In addition and independently, the longitudinal size (along the velocity vector) of each injection has been evaluated from the duration of its crossing by one Cluster spacecraft (Table 2, column 4), giving values between 0.6 and $2.0 R_{E}$. These values are coherent with previously published values obtained by other methods (e.g. Owen et al., 2001; Bosqued et al., 2001).

\subsection{Detailed shape of injection flux tubes}

This event also offers the possibility to study the shape of the injection flux tubes seen both at Cluster and at TC-2. It has been shown by Marchaudon et al. (2006) that the discrimination between planar and tubular structures can be tested for each spacecraft by a variance analysis of the transverse component of the magnetic signal. This discrimination is also essential in the determination of the parallel current from a single-spacecraft (see Sect. 5.1). A planar front results in a linear polarisation of the magnetic signal and is revealed by a low value of the ratio of the intermediate to the largest eigenvalues of the covariance matrix of the signal (variance ratio $r$ ) and by a stable direction of the eigenvector associated with the largest eigenvalue of this covariance matrix (polarisation vector $\boldsymbol{v}$ ).

Figure 7 displays the variance ratio $r$ for each Cluster spacecraft and for two time intervals, one just before the nominal conjunction (period 15:02-15:14 UT including 


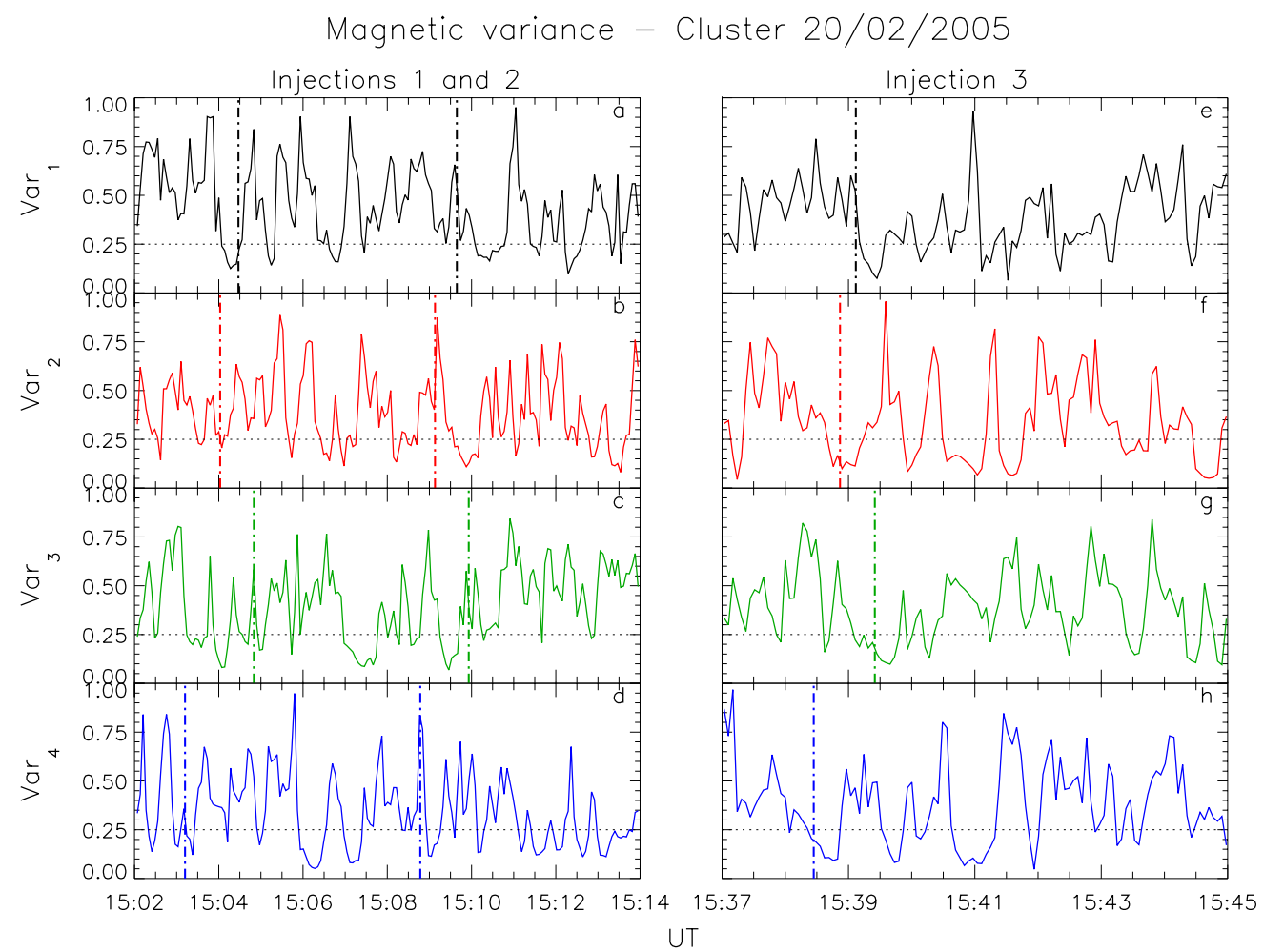

Fig. 7. FGM variance ratio between the intermediate and largest eigenvalues of the covariance matrix of the magnetic signal for each Cluster spacecraft (black line for sc-1, red for sc-2, green for sc-3 and blue for sc-4). (a) to (d) for the 15:02-15:14 UT period (Injections 1 and 2). (e) to (h) for the 15:37-15:45 UT period (Injection 3).

Injections 1 and 2 on the left-hand side) and one just after (period 15:37-15:45 UT including Injection 3 on the righthand side). Similarly, panels (a) and (e) of Fig. 8 display the variance ratio $r$ measured at TC-2 for Injections 1 and 2, and Injection 3 respectively. Although the ratio $r$ is quite variable, indicating a variety of structures, it shows several short periods when $r$ is below 0.25 . These periods are accompanied by a stable polarisation vector $v$ (not shown). Such periods occur in particular around the beginning of each injection seen at Cluster and at TC-2, as defined with PEACE electron fluxes (dash-dotted lines on Figs. 7 and 8). The results are clear especially for Injections 2 and 3, confirming quasi-planar current structures and validating the quasiplanar assumption used in the four-spacecraft timing analysis (Sect. 4.1).

In the variance analysis at each of the four Cluster, when the polarisation is quasi-linear, the polarisation vector $v$ gives locally the direction of the front of the structure. However, these directions differ slightly at the different spacecraft. By fitting these directions with a circle in the plane perpendicular to the magnetic field, the radius of curvature of the front can be determined. Figure 9a illustrates the result of this exercise for the beginning of Injection 3, indicating a concave forward front of the structure, with a radius of $0.38 R_{E}$. The concave shape of the forward front suggests a bean-like structure of the blob. Such an analysis which has been possible for the forward front revealed totally impossible for the backward side of the structure because it was not possible to identify safely any similar features on the four spacecraft (high $r$ and variable $\boldsymbol{v}$ ). This suggests a backward side of the blobs more turbulent than their front side. A sketch of a possible transverse shape of the plasma blob is illustrated in Fig. 9b. For the first time, the detailed shape of an injection flux tube was described, with a well defined front and a more turbulent backward side. This asymmetry between the two sides may be the result of an instability of the backward side of the convecting flux tube (Keskinen and Ossakow, 1983), but may also result from the time history of the injection itself, leading to a different structure of its front and backward sides. For Injections 1 and 2, a well defined front and a turbulent backward side are still visible, but the shape analysis could not be applied due to their smaller extension.

\section{Field-aligned currents in injections flux tubes}

At Cluster, we have tested and compared the results of different methods to calculate FACs: first from magnetic data by the curlometer technique and also by the single-spacecraft method, and then from particle moments. At TC-2, only the 


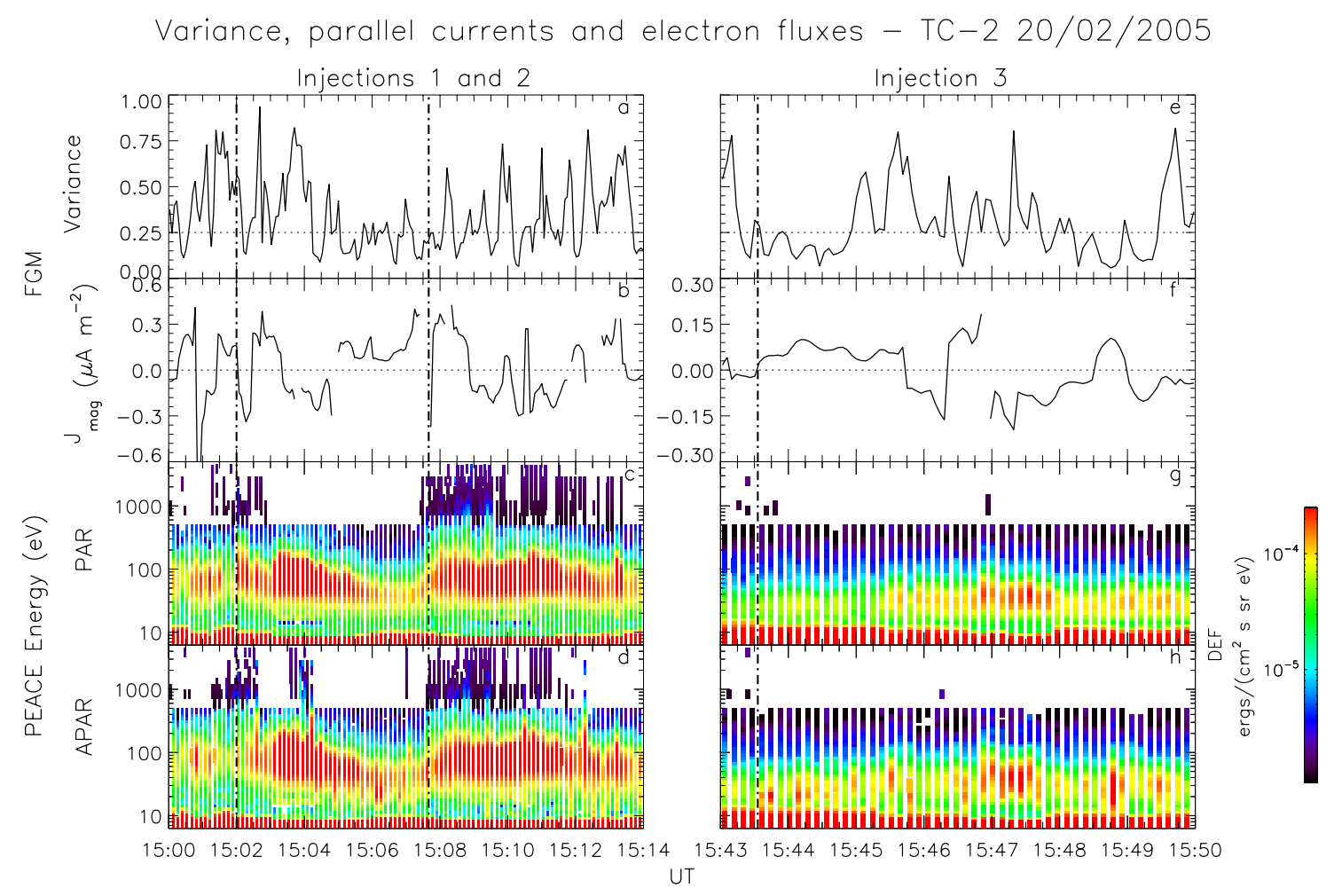

Fig. 8. TC-2 FGM variance ratio $r$ and field-aligned currents deduced from the single-spacecraft method with zero sheet velocity (upward currents are positive), and TC-2 PEACE energy-time electron spectrograms in the parallel and anti-parallel directions. (a) to (d) for the 15:00-15:14 UT period (Injections 1 and 2). (e) to (h) for the 15:43-15:50 UT period (Injection 3).

single-spacecraft method is usable, but it allows to compare FACs at different altitudes.

\subsection{Calculation methods}

With Cluster, it is possible to calculate the current density over the spacecraft tetrahedron volume from Ampere's law. This method, called curlometer, is presented e.g. in Robert et al. (1998) and Dunlop et al. (2002) and consists in calculating the projection of the current density on the normal to each face of the tetrahedron and thus to reconstruct the average current over the spacecraft volume. This method is accurate only if the tetrahedron configuration is regular. In our case, the tetrahedron configuration is almost perfectly regular and although the spacecraft separation is large $(\sim 1000 \mathrm{~km})$, it remains always smaller than the transverse size of the injections $\left(\sim 0.6-2 R_{E}\right)$, thus allowing these injections to be seen almost simultaneously by the four spacecraft. For the curlometer, uncertainties have been estimated by comparing the total current densities calculated by choosing a different reference spacecraft. In our case, two different calculations show similar profiles confirming the stability of the method and the amplitude variations between the two indicates a precision of $\sim 35 \%$ on the current density.
With only one spacecraft, curl b in Ampere's law cannot be calculated safely. However, an estimation of the fieldaligned currents is still possible by assuming that the currents are distributed in infinite parallel sheets, reducing curl $\mathbf{b}$ to a gradient along the normal to the current sheet. This method fails if the previous assumption is not valid, or if the satellite orbit is parallel to the current sheet. No uncertainty can be calculated with this method. However, as stated in Sect. 4.4, the variance ratio $r$ and the polarisation vector $v$ deduced from the covariance matrix of the magnetic signal (Marchaudon et al., 2006) allows to identify planar current structures: if the polarisation vector $v$ is stable and the variance ratio $r$ is low (less than 0.25), then the current structure is planar and the single-spacecraft method is valid. An even more important problem with this method is to estimate the current sheet velocity (equivalent to the velocity of the flux tube) and to take it into account in the FAC calculation. At low (ionospheric) altitudes, this velocity can be neglected (compared with the spacecraft velocity), contrary to mid- or high-altitude, where plasma injections can move much faster than the spacecraft motion. Forgetting this velocity in the FAC calculation can give erroneous current polarity and/or intensity, as shown theoretically by Lockwood et al. (2001a) and observationally with Cluster (Bosqued et al., 2005; Marchaudon et al., 2006) and with the ST-5 mission (Slavin et 


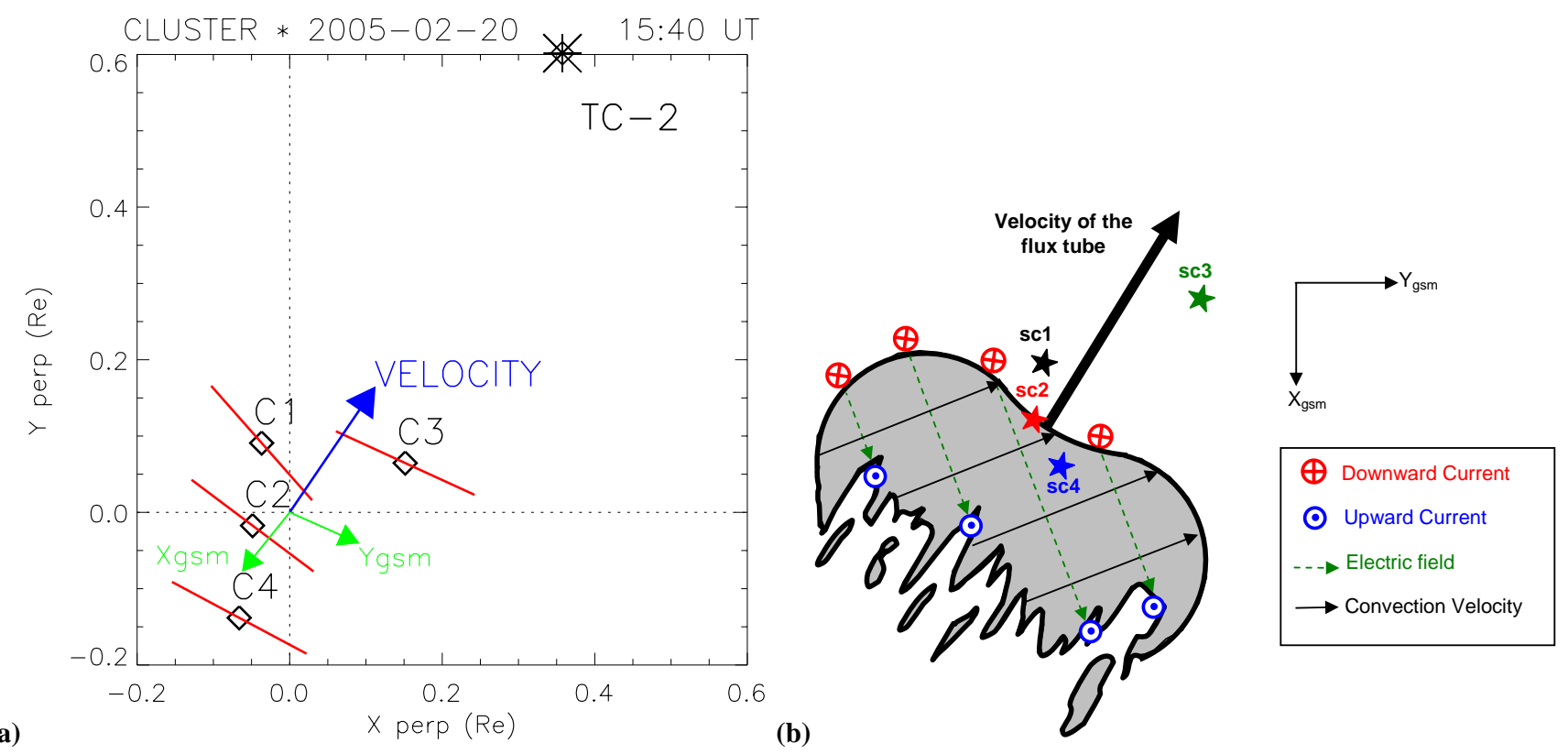

Fig. 9. (a) Polarisation direction at each Cluster spacecraft at the beginning of Injection 3 (15:40 UT) in the plane perpendicular to the magnetic field. The relative position of TC-2 is indicated by a star/diamond symbol, with the centre of mass of the Cluster tetrahedron at the origin. The $X$ and $Y$ axis of the GSM frame have been projected onto this plane (green arrows). The blue arrow represents the velocity of Injection $3(\sim 15: 40 \mathrm{UT})$. (b) Sketch of the transverse shape of Injection 3 deduced from the magnetic variance analysis, and of the electrodynamics of the structure. The black arrows represent the convection velocity, the bold black arrow represents the velocity of the flux tube, the green dashed arrows represents the convection electric field. The blue dots and red crosses represent upward and downward FACs.

al., 2008). For the present case, FACs have been calculated over 7 points measurement, in order to get a smoothing comparable to the curlometer method and only where the sheet direction is not parallel to the satellite orbit (data gaps), and initially with zero sheet velocity. In a further step, these FAC values have been corrected with the velocity of the flux tube obtained from the four-spacecraft timing, directly at Cluster and mapped at the right altitude for TC-2.

An important difference between the curlometer and the single-spacecraft methods is that the former gives a time resolution equal to the data sampling, while the latter which is based on a sequence of measurements, provides temporal smoothing. Both are equivalent only for stationary convecting current sheets evaluated with the same spatial resolution.

Finally, field-aligned currents can also be obtained from particle measurements. With Cluster in burst mode, accurate electron and ion densities and velocities can be calculated from 3-D distribution. Examples of ion and electron FACs in the mid-altitude cusp and their associated uncertainties ( $\sim 35-40 \%$ for electrons and $\sim 40-50 \%$ for ions) have been reported in Marchaudon et al. (2006).

\subsection{Observations}

\subsubsection{At Cluster}

For each injection, we compare the Cluster FACs deduced from the curlometer technique with those from the singlespacecraft method with zero sheet velocity, applied to each satellite. Figure 10 shows FACs calculated by both methods for Injections 1 and 2 (period 15:02-15:14 UT) on the left-hand side and for Injection 3 (period 15:37-15:45 UT) on the right-hand side. Upward currents are positive and downward currents are negative. The beginning of each injection determined from PEACE electron fluxes is marked by a vertical dash-dotted line. We have chosen an arbitrary zero velocity of the flux tubes in the single-spacecraft calculations, because the velocity is different for each injection (see Sect. 4.1) and does not necessarily remain constant, making it difficult to plot accurate currents during a long time interval. The global current profiles obtained by the curlometer and by the four single-spacecraft calculations are remarkably similar. The comparison is better at the beginning of the injections where, as indicated by the variance analysis reported in Sect. 4.4, the infinite current sheet assumption is more valid (low variance ratio $r$ and stable polarisation vector $\boldsymbol{v}$ ). As described in detail below, the profiles show principally for 
Parallel currents - Cluster 20/02/2005

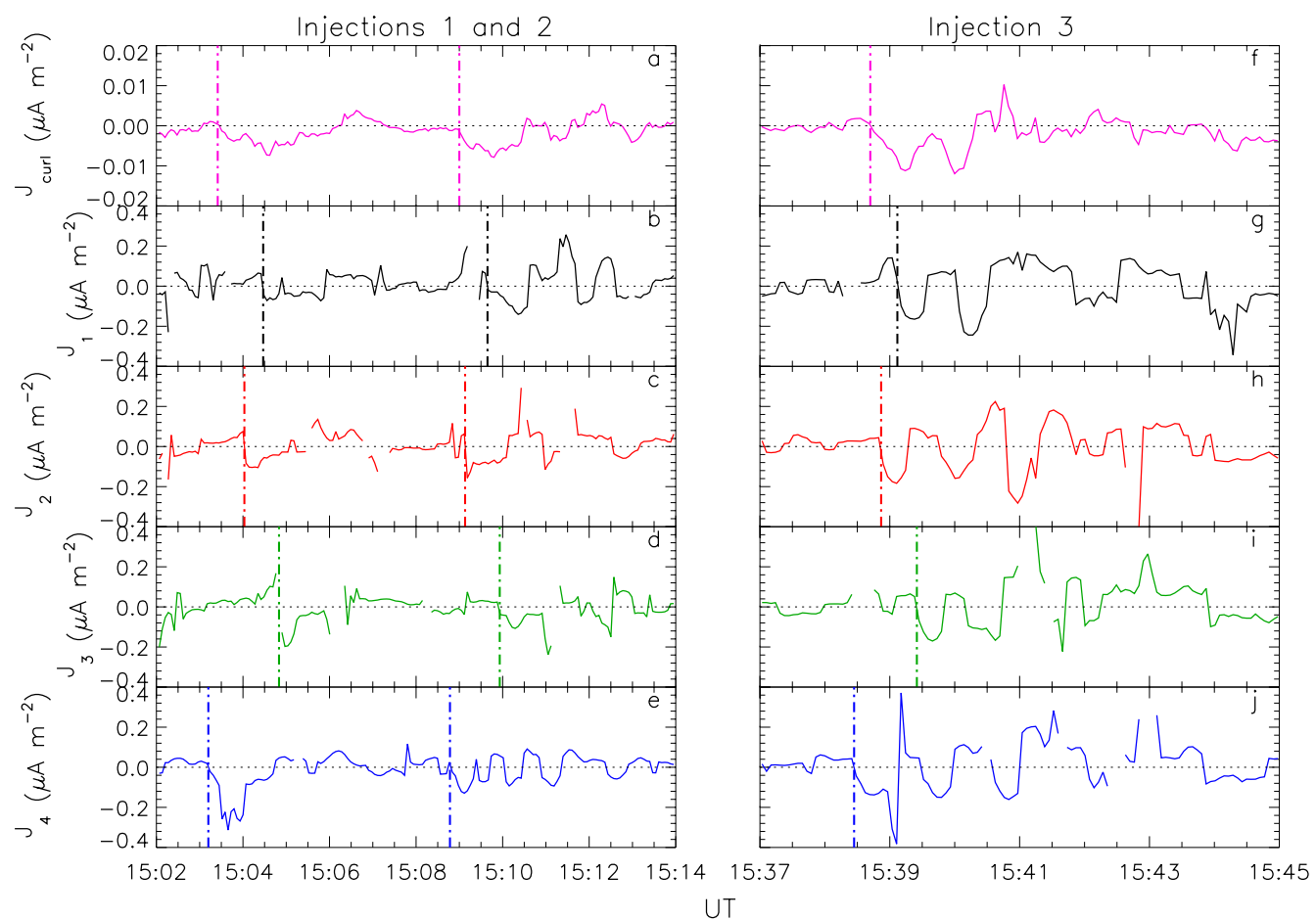

Fig. 10. FGM field-aligned currents calculated with the curlometer method (pink line) and with the single-spacecraft method with zero sheet velocity (black line for sc-1, red for sc-2, green for sc-3 and blue for sc-4). Upward currents are positive. (a) to (e) for the 15:02-15:14 UT period (Injections 1 and 2). (f) to (j) for the 15:37-15:45 UT period (Injection 3).

each injection a pair of downward/upward FACs, despite different current extensions, intensities and the existence of substructures. The curlometer profile which assumes a constant current over the tetrahedral volume is smoother and shows fewer sub-structures.

The current structure of Injection 1 is the simplest with all current calculations showing a pair of downward/upward FACs. The current structure of Injection 2 shows also this pair of downward/upward FACs, with however an upward/downward FACs pair embedded in it, clearly seen on sc-1 and sc-2, smoothed on sc-3 and the curlometer (15:10:30-15:11:30 UT, curlometer time) and split in two successive small upward/downward pairs of FACs on sc-4. Finally, Injection 3 has the most complex structure, with two successive pairs of downward/upward FACs (15:38:35 to $15: 40: 55$ and $15: 40: 55$ to $15: 42: 45 \mathrm{UT}$, curlometer time). Moreover, the two strong downward currents embedded in the first pair of FACs are clearly separated on each of the four spacecraft, but collapse into a single negative current with two distinct peaks with the curlometer (15:38:3515:40:25 UT). A third downward current is also recorded by sc-2 and sc-4 around 15:41 UT. To summarize, a majority of the current sub-structures can be recognised on each of the four spacecraft and even for some of them on the curlometer current, which imply that their extension remains of the order or larger than the Cluster separation.
A remaining problem is the arbitrary zero velocity of the flux tubes (or sheet velocity $V_{s h}$ ) chosen in the singlespacecraft calculations which leads to an erroneous scaling of the current by this method. Here, the Cluster velocity component normal to the current sheet plane $V_{s c}\left(\sim 2.6 \mathrm{~km} \mathrm{~s}^{-1}\right)$ is opposite in direction and about 10 times smaller than the velocity component normal to the sheet $V_{s h}$ in each injection. It results in a velocity of the observer relative to the sheet $\left(V_{s c}-V_{s h}\right)$ in the same direction as the Cluster velocity $V_{s c}$, but much larger. Consequently, the currents calculated with a zero sheet velocity have the right polarity but an overestimated intensity. From Fig. 10, this is clearly the case. All profiles are similar, as discussed earlier, but with a strong difference between intensities given by the curlometer and the single-spacecraft methods, with curlometer currents systematically smaller. For each injection, we estimate the ratio between the currents calculated by the singlespacecraft method with zero sheet velocity and by the curlometer: $J_{/ /}\left(1\right.$-sat, $\left.V_{s h}=0\right) / J_{/ /}($curl) and compare it with the ratio between the currents calculated by the single-spacecraft method with zero sheet velocity and with the velocity of the flux tube calculated by the four-spacecraft timing method (Sect. 4.1): $J_{/ /}\left(1\right.$-sat, $\left.V_{s h}=0\right) / J_{/ /}\left(1-\mathrm{sat}, V_{s h} \neq 0\right)$. This last ratio is equivalent to the ratio $\left(V_{s c}-V_{s h}\right) / V_{s c}$ which can be evaluated independently. The results are presented in Table 3 and show that the two ratios $J_{/ /}\left(1\right.$-sat, $\left.V_{s h}=0\right) / J_{/ /}($curl $)$and 
$J_{/ /}\left(1\right.$-sat, $\left.V_{s h}=0\right) / J_{/ /}\left(1\right.$-sat, $\left.V_{s h} \neq 0\right)$ are fairly close. Since the curlometer current is supposed to be accurate, it confirms the validity of the single-spacecraft method once the right sheet velocity is included. The fact that the ratio $J_{/ /}(1-$ sat, $\left.V_{s h}=0\right) / J_{/ /}$(curl) is however slightly larger than the ratio $J_{/ /}\left(1\right.$-sat, $\left.V_{s h}=0\right) / J_{/ /}\left(1\right.$-sat, $\left.V_{s h} \neq 0\right)$ can be explained again by the smoothing of the curlometer current over the tetrahedral volume. The real peak intensity of the FACs at Cluster is then $\sim 0.01-0.015 \mu \mathrm{A} \mathrm{m}^{-2}$ for Injections 1 and 2 and $\sim 0.01-$ $0.012 \mu \mathrm{A} \mathrm{m}^{-2}$ for Injection 3 .

Finally, we have also calculated ion and electron fieldaligned currents at Cluster from the 3-D particle distributions measured by CIS and PEACE (not shown). The ion current is upward between 0.1 and $0.3 \mu \mathrm{A} \mathrm{m}^{-2}$ inside the injections, and carried by upward ions in the $100-1000 \mathrm{eV}$ energy range. The electron current is downward between -0.1 and $-0.3 \mu \mathrm{A} \mathrm{m}^{-2}$ inside the injections, and carried by upward electrons in the $20-200 \mathrm{eV}$ energy range. These two opposite currents are of the same order, leading to a smaller total current, in agreement with magnetic calculations. Because the electron and ion currents suffer large uncertainties: $\sim 40 \%$ (Marchaudon et al., 2006), and compensate for a large part, a quantitative comparison (profile and intensity) between magnetic and total particles FACs is impossible.

\subsubsection{At TC-2}

For each injection, magnetic FACs at TC-2 are also calculated by the single-spacecraft method with zero sheet velocity. On Fig. 8, panel (b) shows TC-2 field-aligned currents for Injections 1 and 2 (15:00-15:14 UT) and panel (f) for Injection 3 (15:43-15:50 UT). Again, upward currents are positive and downward currents are negative. The beginning of each injection determined from PEACE electron fluxes is marked by a vertical dash-dotted line. The global current profile of each of these 3 injections shows a pair of upward/downward FACs. A localised downward current is observed at the very beginning of Injection 1 and several pairs of small-scale FACs are embedded in the main pair of FACs for Injections 2 and 3.

The polarity of the main pair of FACs is opposite at TC2 and at Cluster for the 3 injections and the TC- 2 current intensity is larger than at Cluster, even if we take into account the field line convergence between both altitudes. Moreover, the comparison at TC-2 between magnetic FACs and PEACE electron fluxes shows that upward currents are associated with dominating upward electron fluxes. This is particularly clear for the periods $15: 12-15: 14$ UT, 15:43:30-15:45:45 UT. These apparently inconsistent results are caused again by the arbitrary zero sheet velocity used in the single-spacecraft calculation which leads in this case to an erroneous estimation of the current polarity. Here, the TC-2 velocity component normal to the current sheet plane $V_{s c}\left(\sim 1.5 \mathrm{~km} \mathrm{~s}^{-1}\right)$ is in the same direction but about 10 times smaller than the velocity component normal to the sheet $V_{s h}$ in each injection. It results in a velocity of the observer relative to the sheet $\left(V_{s c}-V_{s h}\right)$ opposite to the TC-2 velocity $V_{s c}$. Consequently, the currents calculated with a zero velocity of the injections have a wrong polarity in addition to an incorrect intensity. We calculate again the ratio between the currents calculated by the single-spacecraft method with zero sheet velocity and with the velocity of the flux tube calculated by the four-spacecraft timing method at Cluster and mapped at TC-2 altitude along magnetic field lines: $J_{/ /}(1-$ sat, $\left.V_{s h}=0\right) / J_{/ /}\left(1-\right.$ sat, $\left.V_{s h} \neq 0\right)$ (equivalent to the analytic ratio $\left.\left(V_{s c}-V_{s h}\right) / V_{s c}\right)$. The results are shown in the last two columns of Table 3. This ratio is negative for each injection, confirming the wrong polarity of the currents calculated with zero sheet velocity. The real FACs at TC-2 have opposite signs to those plotted on Fig. 8 and in consequence, the main pair of FACs of each injection at TC-2 is downward/upward as for Cluster. In terms of intensity, once this ratio is taken into account in the current calculation, the real peak intensity of the FACs at TC- 2 is $\sim 0.035 \mu \mathrm{A} \mathrm{m}^{-2}$ for Injections 1 and 2 and $\sim 0.012 \mu \mathrm{A} \mathrm{m}^{-2}$ for Injection 3 .

\subsection{Discussion}

\subsubsection{Validity of calculation methods}

We have made the first quantitative comparison of FACs at Cluster calculated by two different methods: the curlometer technique and the single-spacecraft method assuming infinite parallel current sheets. In the previous section, we have shown that the global current profiles and intensities are remarkably similar for the three injections, if the velocity of the current structure is integrated in the single-spacecraft calculation. More surprisingly, the comparison remains satisfactory even if the variance ratio is not always below 0.25 for FACs obtained with the single-spacecraft method. This result means that the threshold at 0.25 for this variance ratio is probably too restrictive and can be increased. It also shows that the two different methods work equally well and gives special credit to the single-spacecraft method, whose validity has rarely been verified.

\subsubsection{Comparison between Cluster and TC-2}

We also realised the first quantitative comparison of FACs at two different altitudes in the magnetosphere with remarkably consistent results.

In terms of structure, the main downward/upward pair of FACs is well identified for each injection at Cluster and at TC-2. Even several small-scale structures embedded inside the injections at TC-2 and at Cluster appear correlated. Thus, the sub-structures of Injections 2 and 3 show similar profiles at TC-2 and Cluster-1. However, the small upward current at the beginning of Injection 1 observed at TC-2 (15:02:15 UT, in real polarity) is not seen at Cluster. 
Table 3. Velocities of the injections at Cluster and TC-2, ratio between FACs calculated without and with sheet velocity and ratio between FACs calculated without sheet velocity and from the curlometer.

\begin{tabular}{|c|c|c|c|c|c|}
\hline Event (Cluster time, UT) & $\begin{array}{c}V_{s h} \\
\left(\mathrm{~km} \mathrm{~s}^{-1}\right)\end{array}$ & $\begin{array}{c}\text { Cluster } \\
J_{/ /\left(1-\text { sat }, V_{s h}=0\right) /} \\
J_{/ /\left(1-\text { sat, }, V_{s h} \neq 0\right)}\end{array}$ & $\begin{array}{c}J_{/ /}\left(1-\text { sat }, V_{s h}=0\right) / \\
J_{/ /}(\text {curl })\end{array}$ & $\begin{array}{c}V_{s h} \\
\left(\mathrm{~km} \mathrm{~s}^{-1}\right)\end{array}$ & 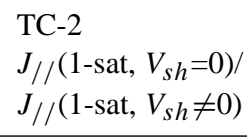 \\
\hline Injection 1 (15:05) & 26.2 & $\sim 10$ & $12-15$ & 14.0 & $\sim-7$ \\
\hline Injection $2(15: 10)$ & 21.5 & $\sim 9$ & $12-15$ & 11.9 & $\sim-6$ \\
\hline Injection 3 (15:39) & 39.4 & $\sim 16$ & $18-20$ & 17.6 & $\sim-13$ \\
\hline
\end{tabular}

In terms of intensity, the currents at TC-2 are between two and three times larger than at Cluster for Injections 1 and 2. This is consistent with current conservation in converging magnetic field lines. On the other hand, TC-2 and Cluster currents have the same intensity in Injection 3. But PEACE spectrograms on Fig. 2 show clearly that TC-2 electron fluxes are also smaller than Cluster electron fluxes, suggesting that Cluster crosses deeper inside Injection 3 while TC-2 crosses more its edge. This can explain the relatively weak current intensity measured by TC- 2 for this injection.

\subsubsection{FAC pattern and particle carriers}

A main pair of FACs, upward at low-latitude and downward at high-latitude, is well identified in each injection at Cluster and at TC-2. This result agrees with the LCC/HCC distribution described by Taguchi et al. (1993) and the C1/C2 distribution described by e.g Sandholt and Farrugia (2007a, b) for negative IMF- $B_{y}$ periods, in which a pair of opposite parallel currents flows on each side of an old reconnected flux tube tailward of the polar cusp. However, the observation of small-scale sub-structures by Cluster and TC-2 in the injections shows that their internal structure can be more complex than a simple current pair as proposed in the $\mathrm{C} 1 / \mathrm{C} 2$ and LCC/HCC models and could result from the turbulent downstream side of these injections. We have shown (see Sect. 4.1.2 above) that the plasma convection inside each injection (duskward and antisunward) differs significantly from the velocities of the injection flux tubes (almost purely antisunward). This difference allows to explain the polarity of the current pair, first downward then upward observed, by TC- 2 and Cluster, as illustrated on the bean-shaped flux tube schematic of Fig. 9b. Because ionospheric observations are too sparse, it was not possible to draw a complete electrodynamic picture of these injections and to check whether they are isolated structures with ionospheric closure of parallel currents occurring totally inside the flux tubes.

From Cluster data, we show that the parallel currents result from a small difference between upward electron and ion fluxes, implying a net upward flow of the particles inside the injections. In general, downward flow is expected inside magnetosheath injections. However, as the injections observed here are fossil signatures observed in the plasma mantle downstream of the cusp (Lockwood et al., 2001b; Lund et al., 2008), their motion is governed mainly by the magnetosheath flow and a majority of ions of magnetosheath origin has already mirrored (net upward flow). How the associated electron population with such high energies can be maintained in these injections (they should have already escaped the magnetosphere) is still an open question, but is beyond the scope of this paper.

\section{Conclusions}

Thanks to a conjunction between Cluster, TC-2 and SuperDARN close to the northern polar cusp, we determine the velocity, the transverse sizes, perpendicular and parallel to this velocity, and the shape of three magnetic flux tubes of magnetosheath plasma injection. The velocity of the flux tubes determined by the Cluster four-spacecraft timing analysis, is almost purely antisunward, in contrast with the antisunward and duskward convection velocity inside the flux tubes. The transverse sizes are defined from the Cluster-TC-2 separation perpendicular to the magnetic field, and from the time spent by a Cluster spacecraft in the structure; they are comprised between 0.6 and $2 R_{E}$ in agreement with previous studies. Finally, using a comparison of the eigenvectors deduced from a variance analysis of the magnetic perturbation at the four Cluster and at TC-2, we show that the upstream side of the injection flux tubes is magnetically well defined, with even a concave front for the third one giving a bean-like shape, whereas the downstream side is far more turbulent. We also realise the first quantitative comparison of field-aligned currents at Cluster calculated from the curlometer technique and from the single-spacecraft method, assuming infinite parallel current sheets and taking into account the velocity of the plasma injection flux tubes. The consistency between the two results confirms the validity of both calculation methods. Finally, we compare the field-aligned currents distribution of the three injection flux tubes at two different altitudes, Cluster and TC-2. The profiles look similar, with mainly a pair of opposite field-aligned currents, upward at low-latitude and downward at high-latitude. In terms of intensity, the FACs calculated at Cluster are two to three times 
less intense than at TC-2, in agreement with magnetic field line convergence, apart for the third flux tube, where the intensity is similar. This can be explained by the fact that TC-2 crosses the edge of the third tube. Finally, we calculate the ion and electron currents from Cluster particle moments and show that the FACs are carried by upward ions and electrons.

Acknowledgements. The authors thank the PIs I. Dandouras, E. Lucek and T. Zhang for providing the CIS Cluster, the FGM Cluster and FGM TC-2 data, respectively. The authors acknowledge the PIs N. Ness and D. J. McComas, and the ACE Science Center for providing the ACE data.

Topical Editor I. A. Daglis thanks G. Paschmann and two other anonymous referees for their help in evaluating this paper.

\section{References}

Balogh, A., Carr, C. M., Acuña, M. H., et al.: The Cluster Magnetic Field Investigation: overview of in-flight performance and initial results, Ann. Geophys., 19, 1207-1217, 2001, http://www.ann-geophys.net/19/1207/2001/.

Basinska, E. M., Burke, W. J., and Heinemann, M. A.: A user's guide to locating flux transfer events in low-altitude satellite measurements - An S3-2 case study, J. Geophys. Res., 94, 6681$6691,1989$.

Bosqued, J. M., Phan, T. D., Dandouras, I., et al.: Cluster observations of the high-latitude magnetopause and cusp: initial results from the CIS ion instruments, Ann. Geophys., 19, 1545-1566, 2001, http://www.ann-geophys.net/19/1545/2001/.

Bosqued, J.-M., Escoubet, C. P., Frey, H. U., Dunlop, M., Berchem, J., Marchaudon, A., Cerisier, J.-C., Fazerkerley, A., Budnik, E., Lavraud, B., Rème, H., Laakso, H., and Balogh, A.: Multipoint observations of transient reconnection signatures in the cusp precipitation: A CLUSTER-IMAGE detailed case study, J. Geophys. Res., 110, A03219, doi:10.1029/2004JA010621, 2005.

Carr, C., Brown, P., Zhang, T. L., et al.: The Double Star magnetic field investigation: instrument design, performance and highlights of the first year's observations, Ann. Geophys., 23, 27132732, 2005, http://www.ann-geophys.net/23/2713/2005/.

Cerisier, J.-C., Marchaudon, A., Bosqued, J.-M., McWilliams, K., Frey, H., Bouhram, M., Laakso, H., Dunlop, M., Förster, M., and Fazakerley, A.: Plasma injections and flow bursts in the dayside cusp triggered by solar wind pressure pulses, J. Geophys. Res., 110, A08204, doi:10.1029/2004JA010962, 2005.

Cowley, S. W. H. and Lockwood, M.: Excitation and decay of solar wind-driven flows in the magnetosphere-ionosphere system, Ann. Geophys., 10, 103-115, 1992.

Dunlop, M. W., Balogh, A., Glassmeier, K.-H., and Robert, P.: Four-point Cluster application of magnetic field analysis tools: The Curlometer, J. Geophys. Res., 107(A11), 1384, doi:10.1029/2001JA005088, 2002.

Eastman, T. E., Hones Jr., E. W., Bame, S. J., and Asbridge, J. R.: The magnetospheric boundary layer - Site of plasma, momentum and energy transfer from the magnetosheath into the magnetosphere, Geophys. Res. Lett., 3, 685-688, 1976.

Escoubet, C. P., Smith, M. F., Fung, S. F., Anderson, P. C., Hoffman, R. A., Basinska, E. M., and Bosqued, J.-M.: Staircase ion signature in the polar cusp-A case study, Geophys. Res. Lett., 19, 1735-1738, 1992.
Farrugia, C. J., Lund, E. J., Sandholt, P. E., Wild, J. A., Cowley, S. W. H., Balogh, A., Mouikis, C., Möbius, E., Dunlop, M. W., Bosqued, J.-M., Carlson, C. W., Parks, G. K., Cerisier, J.C., Kelly, J. D., Sauvaud, J.-A., and Rème, H.: Pulsed flows at the high-altitude cusp poleward boundary, and associated ionospheric convection and particle signatures, during a Cluster FAST - SuperDARN- Søndrestrøm conjunction under a southwest IMF, Ann. Geophys., 22, 2891-2905, 2004, http://www.ann-geophys.net/22/2891/2004/.

Fazakerley, A. N., Carter, P. J., Watson, G., et al.: The Double Star Plasma Electron and Current Experiment, Ann. Geophys., 23, 2733-2756, 2005, http://www.ann-geophys.net/23/2733/2005/.

Greenwald, R. A., Baker, K. B., Dudeney, J. R., et al.: Darn/SuperDARN: a global view of the dynamics of highlatitude convection, Space Sci. Rev., 71, 761-796, 1995.

Hasegawa, H., Sonnerup, B. U. Ö., Owen, C. J., Klecker, B., Paschmann, G., Balogh, A., and Rème, H.: The structure of flux transfer events recovered from Cluster data, Ann. Geophys., 24, 603-618, 2006, http://www.ann-geophys.net/24/603/2006/.

Johnstone, A. D., Alsop, C., Burge, S., et al.: PEACE: a Plasma electron and current experiment, Space Sci. Rev., 79, 351-398, 1997.

Keskinen, M. J. and Ossakow, S. L.: Theories of high-latitude ionospheric irregularities: a review, Radio Sci., 18, 1077-1091, 1983.

Kullen, A., Buchert, S., Karlsson, T., Johansson, T., Lileo, S., Eriksson, A., Nilsson, H., Marchaudon, A., and Fazakerley, A. N.: Plasma transport along discrete auroral arcs and its contribution to the ionospheric plasma convection, Ann. Geophys., 26, 32793293, 2008, http://www.ann-geophys.net/26/3279/2008/.

Lavraud, B., Fedorov, A., Budnik, E., Thomsen, M. F., Grigoriev, A., Cargill, P. J., Dunlop, M. W., Rème, H., Dandouras, I., and Balogh, A.: High-altitude cusp flow dependence on IMF orientation: A 3-year Cluster, statistical study, J. Geophys. Res., 110, A02209, doi:10.1029/2004JA010804, 2005.

Lee, L. C.: Magnetic flux transfer at the Earth's magnetopause, in: Solar wind magnetosphere coupling, edited by: Kamide, Y. and Slavin, J. A., pp. 297-314, Terra Scientific, Tokyo, 1986.

Lockwood, M., Cowley, S. W. H., Sandholt, P. E., and Lepping, R. P.: The ionospheric signatures of flux transfer events and solar wind dynamic pressure changes, J. Geophys. Res., 95, 1711317135, 1990.

Lockwood, M., Milan, S. E., Onsager, T., Perry, C. H., Scudder, J. A., Russell, C. T., and Brittnacher, M.: Cusp ion steps, fieldaligned currents and poleward moving auroral forms, J. Geophys. Res., 106, 29555-29569, 2001a.

Lockwood, M., Opgenoorth, H., van Eyken, A. P., et al.: Coordinated Cluster, ground-based instrumentation and low-altitude satellite observations of transient poleward-moving events in the ionosphere and in the tail lobe, Ann. Geophys., 19, 1589-1612, 2001b, http://www.ann-geophys.net/19/1589/2001/.

Lund, E. J., Farrugia, C. J., and Sandholt, P. E.: Momentum transfer at the high-latitude magnetopause and boundary layers, Ann. Geophys., 26, 2449-2458, 2008, http://www.ann-geophys.net/26/2449/2008/.

Marchaudon, A., Cerisier, J.-C., Bosqued, J.-M., Owen, C. J., Fazakerley, A. N., and Lahiff, A. D.: On the structure of field-aligned currents in the mid-altitude cusp, Ann. Geophys., 24, 33913401, 2006, http://www.ann-geophys.net/24/3391/2006/.

Marchaudon, A., Cerisier, J.-C., Bosqued, J.-M., Dunlop, M. W., 
Wild, J. A., Décréau, P. M. E., Förster, M., Fontaine, D., and Laakso, H.: Transient plasma injections in the dayside magnetosphere: one-to-one correlated observations by Cluster and $\mathrm{Su}-$ perDARN, Ann. Geophys., 22, 141-158, 2004a,

http://www.ann-geophys.net/22/141/2004/.

Marchaudon, A., Cerisier, J.-C., Greenwald, R. A., and Sofko, G. J.: Electrodynamics of a flux transfer event: Experimental test of the Southwood model, Geophys. Res. Lett., 31, L09809, doi:10.1029/2004GL019922, 2004b.

Milan, S. E., Lester, M., Cowley, S. W. H., and Brittnacher, M.: Convection and auroral response to a southward turning of the IMF: Polar UVI, CUTLASS, and IMAGE signatures of transient magnetic flux transfer at the magnetopause, J. Geophys. Res., 105, 15741-15755, 2000.

Moen, J., Rinne, Y., Carlson, H. C., Oksavik, K., Fujii, R., and Opgenoorth, H.: On the relationship between thin Birkeland current arcs and reversed flow channels in the winter cusp/cleft ionosphere, J. Geophys. Res., 113, A09220, doi:10.1029/2008JA013061, 2008.

Newell, P. T. and Meng, C.-I.: Mapping the dayside ionosphere to the magnetosphere according to particle precipitation characteristics, Geophys. Res. Lett., 19, 609-612, 1992.

Oksavik, K., Moen, J., and Carlson, H. C.: High-resolution observations of the small-scale flow pattern associated with a poleward moving auroral form in the cusp, Geophys. Res. Lett., 31, L11807, doi:10.1029/2004GL019838, 2004.

Owen, C. J., Fazakerley, A. N., Carter, P. J., Coates, A. J., Krauklis, I. C., Szita, S., Taylor, M. G. G. T., Travnicek, P., Watson, G., Wilson, R. J., Balogh, A., and Dunlop, M. W.: Cluster PEACE observations of electrons during magnetospheric flux transfer events, Ann. Geophys., 19, 1509-1522, 2001, http://www.ann-geophys.net/19/1509/2001/.

Provan, G., Yeoman, T. K., and Milan, S. E.: CUTLASS Finland radar observations of the ionospheric signatures of flux transfer events and the resulting plasma flows, Ann. Geophys., 16, 14111422, 1998, http://www.ann-geophys.net/16/1411/1998/.

Rème, H., Aoustin, C., Bosqued, J. M., et al.: First multispacecraft ion measurements in and near the Earth's magnetosphere with the identical Cluster ion spectrometry (CIS) experiment, Ann. Geophys., 19, 1303-1354, 2001,

http://www.ann-geophys.net/19/1303/2001/.

Rijnbeek, R. P., Cowley, S. W. H., Southwood, D. J., and Russell, C. T.: A survey of dayside flux transfer events observed by ISEE 1 and 2 magnetometers, J. Geophys. Res., 89, 786-800, 1984.

Rinne, Y., Moen, J., Oksavik, K., and Carlson, H. C.: On the occurrence of reversed flow events in the cusp ionosphere observed by European Incoherent Scatter (EISCAT) Svalbard radar, J. Geophys. Res., 112, A10313, doi:10.1029/2007JA012366, 2007.

Robert, P., Dunlop, M. W., Roux, A., and Chanteur, G.: Accuracy of current density determination, in: Analysis Methods for MultiSpacecraft Data, edited by: Paschmann, G. and Daly, P. W., pp. 395-418, ISSI Sci. Rep., SR-001, Kluwer Acad., Norwell, Mass., 1998.

Rosenbauer, H., Grünwaldt, H., Montgomery, M. D., Paschmann, G., and Sckopke, N.: Heos 2 plasma observations in the distant polar magnetosphere: the plasma mantle, J. Geophys. Res., 80, 2723-2737, 1975.
Ruohoniemi, J. M. and Baker, K. B.: Large-scale imaging of highlatitude convection with Super Dual Auroral Radar Network HF radar observations, J. Geophys. Res., 103, 20797-20811, 1998.

Russell, C. T. and Elphic, R. C.: Initial ISEE magnetometer results: magnetopause observations, Space Sci. Rev., 22, 681-715, 1978.

Sandholt, P. E., Lockwood, M., Oguti, T., Cowley, S. W. H., Freeman, K. S. C., Lybekk, B., Egeland, A., and Willis, D. M.: Midday auroral breakup events and related energy and momentum transfer from the magnetosheath, J. Geophys. Res., 95, 10391060, 1990.

Sandholt, P. E., Farrugia, C. J., and Denig, W. F.: Detailed dayside auroral morphology as a function of local time for southeast IMF orientation: implications for solar wind coupling, Ann. Geophys., 22, 3537-3560, 2004,

http://www.ann-geophys.net/22/3537/2004/.

Sandholt, P. E. and Farrugia, C. J.: Poleward moving auroral forms (PMAFs) revisited: responses of aurorae, plasma convection and Birkeland currents in the pre- and postnoon sectors under positive and negative IMF $B_{y}$ conditions, Ann. Geophys., 25, 16291652, 2007a, http://www.ann-geophys.net/25/1629/2007/.

Sandholt, P. E. and Farrugia, C. J.: Role of poleward moving auroral forms in the dawn-dusk auroral precipitation asymmetries induced by IMF By, J. Geophys. Res., 112, A04203, doi:10.1029/2006JA011952, 2007b.

Saunders, M. A., Russell, C. T., and Sckopke, N.: Flux transfer events: scale size and interior structure, Geophys. Res. Lett., 11, 131-134, 1984.

Slavin, J. A., Le, G., Strangeway, R. J., Wang, Y., Boardsen, S. A., Moldwin, M. B., and Spence, H. E.: Space Technology 5 multipoint measurements of near-Earth magnetic fields: Initial results, Geophys. Res. Lett., 35, L02107, doi:10.1029/2007GL031728, 2008.

Sonnerup, B. U. Ö., Hasegawa, H., and Paschmann, G.: Anatomy of a flux transfer event seen by Cluster, Geophys. Res. Lett., 31, L11803, doi:10.1029/2004GL020134, 2004.

Southwood, D. J.: The ionospheric signature of flux transfer events, J. Geophys. Res., 92, 3207-3213, 1987.

Stern, D.: Magnetospheric dynamo processes, in: Magnetospheric Currents, Geophys. Monograph Ser., vol. 28, edited by: Potemra, T., AGU, Washington, D.C., 200-207, 1984.

Taguchi, S., Sugiura, M., Winningham, J. D., and Slavin, J. A.: Characterization of the IMF By-dependent field-aligned currents in the cleft region based on DE 2 observations, J. Geophys. Res., 98, 1393-1407, 1993.

Tsyganenko, N. A.: A model of the near magnetosphere with a dawn-dusk asymmetry: 1. Mathematical structure, J. Geophys. Res., 107(A8), 1179, doi:10.1029/2001JA000219, 2002a.

Tsyganenko, N. A.: A model of the near magnetosphere with a dawn-dusk asymmetry: 2. Parameterization and fitting to observations, J. Geophys. Res., 107(A8), 1176, doi:10.1029/2001JA000220, 2002b.

Vontrat-Reberac, A., Bosqued, J.-M., Taylor, M. G. G. T., Lavraud, B., Fontaine, D., Dunlop, M. W., Laakso, H., CornilleauWerhlin, N., Canu, P., and Fazakerley, A.: Cluster observations of the high-altitude cusp for northward interplanetary magnetic field: A case study, J. Geophys. Res., 108(A9), 1346, doi:10.1029/2002JA009717, 2003. 